80

pare certe forme di animali e di piante iu preferenza di certe altre della medesima classe, per effetto de' mezzi e delle condizioni differenti, nelle quali questi esseri sono stati prodotti e si sono propagati. Questa seconda legge ha più o meno interrotto il piano primitivo generale, producendo spezie di anomalie particolari, le quali pertanto non impediscono ad un occhio accorto di riconoscere il piano generale ch' è dominato nella comparsa degli esseri, al quale piano le anomalie accidentali sono subordinate.

(Sarà continuato)

\title{
Sopra il magnetismo generato dalla corrente elettrica.
}

\section{Osservazioni di C. Matteucci.}

Malgrado i molti lavori fatti sopra questo soggetto di cui l' interesse si rende ogni gionno più grande, noi siamo ancora ben lontani dal conoscerne le leggi fondamentali e dal possederne la teoria.

Per dimostrare questa verita basterebbe di ricordare il numero limitato dei corpi nei quali il magnetismo si sveglia, l'influenza del calore e delle azioni molecolari sul magnetismo, i fatti ben noti di Savary ec. Ho perciò pensato che fosse di qualche interesse pubblicare qualche nuovo fatto relativo al magnetismo generato dalla corrente nel ferro, e all' influenza di certe azioni molecolari sopra di esso.

Ho preso a studiare il magnetismo sviluppato dalla corrente elettrica in una verga di ferro dolce con un metodo assai semplice e che è il solo che può usarsi in certe ricerche. Uno spirale di trenta giri formato con un filo di rame di 3 millimetri di diametro era messo in comunicazione con una pila di venti elementi di Bunsen e serviva a magnetizzare la verga di ferro. Avevo un altra 
spirale a filo sottilissimo di rame, ( un terzo di millimetro ) di 800 giri, i di cui capi erano congiunti con quello di un galvanometro assai sensibile. Ambidue queste spirali avevano lo stesso diametro interno a modo da potervisi introdurre esattamente una verga di ferro dolce che aveva tre centimetri di diametro. Prima di descrivere l'esperienza fatta con questa verga dirò che provai a tenere le due spirali l'una in contatto dell' altra colle loro estremità: chiudendo il circuito della pila ebbi un segno di corrente indotta che fu appena di mezzo grado al mio galvanometro.

La verga di ferro dolce che usai nelle esperienze che descriverò era lunga quattro metri: la spirale magnetizzante era fissa ad una delle estremità della verga, e l'altra spirale era introdotta nell' interno della verga e poteva scorrere sopra di essa trovandosi così a diverse distanze dalla prima spirale. Nella prima esperienza le due spirali in contatto colle loro estremità avevano le loro metà a $0 \mathrm{~m}, 11$ di distanza. Chiudendo il circuito della pila avevo delle correnti indotte di diversa intensità secondo le varie distanze delle spirali. Ecco i risultati ottenuti i quali proveranno come si propaga il magnetismo prodotto dalla corrente elettrica, dal punto su cui questa agisce: onde assicurarmi della costanza della corrente rimettevo finite tutte le esperienze le due spirali in una stessa posizione.

Distanza fra le spirali

a contatto

$0 \mathrm{~m}, 10$

0,20

0,30

0,40

0,50

0,60

0,70

0,80

0,90

1

1,10

1,20

1,30

1,40

cim. an. $V$
Arco di deviazione della cor. indotta

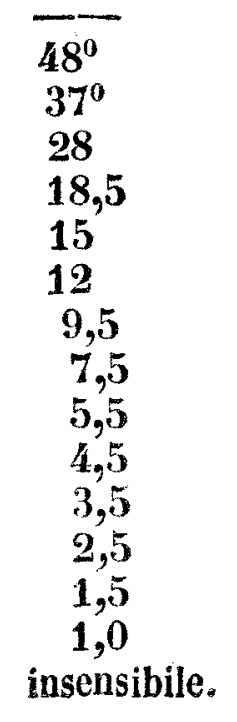


82

Mostra questo quadro come rapidamente decresce lo stato magnetico preso dai vari punti della verga di ferro a delle distanze dalla spirale magnetizzante che vanno sempre crescendo.

Ho paragonato il magnetismo sviluppato da una data corrente secondo che era varia la lunghezza della verga di ferro che rimaneva al difuori della spirale. A questo fine colloco la spirale magnetizzante in una delle estremità della verga in guisa che la spirale indotta in contatto dell' altra può trovarsi ora sopra un pezzo di ferro dolco che è l' estremo della verga, ora sopra un pezzo della verga stessa che si prolunga al di là della spirale.

La differenza del magnetismo generato sui due puntì della verga che sono ad egual distanza dal mezzo della spirale magnetizzante, è assai grande : il magnetismo preso dal pezzo della verga che è presso alla sue estremità è assai minore di quello preso dal pezzo interno della verga. Questo risultato si verifica sopra verghe di diversa lunghezza e diametro. Colla verga sudescritta la corrente indotta $\mathrm{fu}$ in varie esperienze di $48^{\circ}, 50^{\circ}, 45^{\circ}$, allorchè la spirale era ad un estremità, mentre trovandosi nell' interno te deviazioni corrispondenti furono $85^{\circ}, 86^{\circ}$, $84^{\circ}$. Variando questa esperienza a modo che la differenza della lunghezza della verga dalle due parti della spirale magnetizzante fosse or maggiore o minore, si trova la suddetta differenza nel magnetismo generato tanto più grande quanto è più grande questa differenza: ciò però dentro i limiti già indicati della lunghezza della verga a cui si estende il magnetismo generato dalla corrente; di modo chè con una verga simile a quella sudescritta non si riscontra più differenza del magnetismo generato dalle due parti della spirale se da una parte la lunghezza della verga e di $1 \mathrm{~m}, 30$ e dell' altro molti metri.

La posizione della spirale magnetizzante che sviluppa il massimo di un magnetismo in una verga di ferro di una certa lunghezza trovasi alla metà circa della verga. Ecco i numeri trovati in una esperienza fatta con una verga lunga circa 4m. Essendo la spirale indotta ad una estremita della verga in contatto della spirale magnetizzante si aveva una corrente di $35^{\circ}$, mentre mettendo l' indotta dalla parte interna della verga sempre in contatto della spirale magnetizzante si aveva una corrente di $65^{\circ}$. E questo il falto gia descritto. Ripetendo queste stesse sperienze col- 
la spirale magnetizzante in mezzo della verga, l' ago và a $90^{\circ}$.

Un fenomeno di magnetismo generato dalla corrente che si scorge assai distintamente con questo stesso metodo, è quello del tempo finito che impiega il ferro a prendere il massimo di magnetismo sotto una data corrente e della sua conservazione, cessata la corrente. L' esperienza è disposta al solito, cioè intorno al cilindro di ferro, sono la spirale della pila è quella che và al galvanometro. Chiudo il circuito della pila è lasciatolo cosi per un tempo più o meno lungo, riapro questo circuito e chiudo quello dell'altra spirale dopo un tempo variabile quantunque sempre assai piccolo. Ottengo una corrente indotta più o meno grande diretta come avrei ottenuto se avessi aperto nello stesso tempo il circuito della pila, cioè quella del magnetismo che si distrugge. La corrente elettromagnetica indotta, così ottenuta, diminuisce assai rapidamente per poco che si prolunghi l' intervallo di tempo tra l'aprire il circuito della pila e chiudere l' indotto: dopo l'intervallo di un terzo di secondo la corrente indotta era tuttavia sensibile. Ho notato che secondo che il circuito della pila era stato chiuso più o meno lungamente, la corrente indotta che si aveva, chiudendo dopo un intervallo di tempo costante, variava. II massimo effelto s'ottiene allorchè la durata del passaggio della corrente della pila non oltrepassa un secondo, o forse meno; ciò è probabilmente dovuto alla corrente della pila che si va indebolendo tenendo il circuito chiuso. Se poi si chiude il circuito indotto l' istante dopo che il circuito della pila è stato chiuso, si ha ancora in questo caso un circuito indotto che è nel senso del magnetismo che si genera. Anche in questo caso il segno della corrente indotta è ancora sensibile lasciando un intervallo di un terzo di secondo frà la chiusura dei due circuiti.

Queste esperienze provano bene che il massimo del magnetismo generato nel ferro dalla corrente non si ottiene al momento stesso che il circuito è chiuso, e che cessata la corrente seguita il magnetismo per un certo tempo decrescendo sempre.

Mi rimane a descrivere $\mathrm{i}$ resultati ottenuti sottomettendo il ferro nel tempo in cui è soggetto all' azione della corrente sia alla torsione, sia agli urti. Prendo una verga di ferro dolce di $0 \mathrm{~m}, 03$ di diametro, e la fisso stabilmente in un blocco di marmo piantato nel suolo: la soli- 


\section{4}

ta spirale a grosso filo è fissata alla metà della verga mentre l'altra spirale a filo lungo e sottile è in comunicazione col galvanometro e fissata sopra questa. Prima di far passare la corrente della pila ho provato a torcere la verga o a percuoterla con un martello di legno, onde determinare gii effetti avuli in tal caso dalla sola azione magnetica della terra. Con queste azioni meccaniche usate il più fortemente che sia possibile, avevo delle correntí di soli $2^{0}$ o $3^{0}$ al circa, dirette come se la verga si fosse magnetizzata in modo da avere il polo australe nel basso della verga stessa : è questo il fatto già trovato da Gifbert, Scoresby e Pouillet. Dopo questi primi tentativi lasciai la verga in riposo per molte ore e feci passare la corrente di una pila di 20 elementi di Bunsen nella spirale a filo grosso. Lasciato tornare l' ago del galvanometro a zero cominciai ad agire sulla verga sia percuotendola col martello, sia torcendola per mezzo di una lunga asta di legno che con una testa di bronzo era fissata alla sua estremità superiore. Devo qui notare che dopo le torsioni l' asta non ritornava mai alla sua prima posizione rimanendo cosi la verga torta $\mathrm{e}$ tanto più quanto più queste torsioni erano state ripetute.

Il fenomeno costante che si ottiene coi primi colpi o colle prime torsioni fatte subire alla verga è quello di una corrente indotta che prova un aumento nel magnetismo generato nella verga dalla corrente e ciò qualunque sia la sua direzione. Usando la torsione ad ottenere questi effetti la prima ricerca che mi occupò fu quella di stabilire se vi era differenza nell' accrescimento del magnetismo secondo chè la torsione accadeva nel senso stesso della corrente della pila, $\boldsymbol{o}$ in senso contrario. Per esser certo che la torsione non sia permanente, usai in alcune esperienze delle sottili verghe d' acciajo disposte come nell'esperienza precedente. Tentai anche di misurare il magnetismo preso da verghe d' acciajo eguali circondate da spirali simili percorse dall' istessa corrente dopo aver torte queste verghe in direzioni contrarie durante il passaggio della corrente. In ogni esperienza v' era sempre una verga lasciata senza torsione. Ripetei questo stesso esperimento usando la scarica della bottiglia in luogo della corrente. In ogni caso il magnetismo che per l' influenza della torsione si svolgeva o si fissava nel ferro o nell'acciajo fu trovato l' istesso, qualunque fosse il senso della torsione rispetto alla direzione della corrente e sempre maggiore di quello fissato nell' acciajo senza torsione. 
Ecco i fenomeni particolari presentati dal ferro dolce sotto la torsione. Tutte lo volte che esso si torce o si percuote, cresce il magnetismo : cessata la corrente, torto o percosso, il magnetismo si distrugge più rapidamente; nel primo caso si hanno segni di una corrente indotta come al principio del magnetismo e nell' altro di una corrente indotta di magnetismo che cessa. Qualunque verga di ferro che non sia stata troppo lungamente torta - percossa presenta questi fenomeni, e se anche lo fu, basta di lasciarla per alcune ore in riposo perchè questi fenomeni si riproduchino. Seguitando a torcere, od a percuotere una verga di ferro dolce molte volte di seguito e a piccolo intervallo di tempo, i fenomeni sono assai diversi . Cosi seguitando a torcere una verga nel tempo che è magnetizzata dalla corrente, si hanno per tre o quattro torsioni successive delle correnti indotte che provano un aumento di magnetismo, ma che però vanno sempre diminuendo. Ed importa di notare che questo risultalo è lo stesso sia che si torca sempre in un senso sia che si torca alternativamente in senso contrario e sempre della stessa quantità. Ho osservato però nel maggior numero dei casi una differenza fra questi due modi di toreere, se si lasciava un lungo intervallo di tempo, fra una torsione e l'altra : la corrente indotta per una seconda torsione era in questo caso più forte torcendo in senso contrario della prima, di quello che torcendo nello stesso senso .

Continuando a torcere una verga di ferro magnetizzato dalla corrente, dopo i primi tre o quattro colpi che mostrano un aumento sempre decrescente di magnetismo, si cominciano a scorgere dei segni di corrente indotta in senso contrario, cioè di magnetismo che si distrugge. Anche in questo caso i segni della corrente indotta vanno diminuendo e continuando a torcere la verga con colpi successivi, ricompajono di nuovo delle correnti per magnetismo che cresce e al solito per cinque o sei colpi i segni sono sempre nello stesso senso diminuendo costantemente. Ho agito cosi sopra una stessa verga con cinquanta o sessanta colpi di torsione, dati di seguito e a mezzo secondo circa l'uno all' altro. I fenomoni continuarono sempre a presentarsi nello stesso ordine cioè di magnetismo che cresce, che indi cala, per poi diminuire di nuovo e così successivamente.

Cessando di far passare la corrente, allora tutti i 
86

colpi o le torsioni che si danno alla verga sono sempre seguite da diminuzioni di magnetismo che spariscono affatto dopo tre o quattro volte. $\mathbf{E}$ curioso a notarsi che anche molte ore dopo che la verga ha cessato di essere magnetizzata per il passaggio della corrente, alla prima torsione che allora le si dà, si ha una corrente indotta così forte come si sarebbe avuta l'istante dopo che il circuito è stato aperto.

Descriverò infine un'ultima esperienza fatta usando invece di un cilindro di ferro dolce un cilindro di limatura di ferro; consisteva 1' apparecchio in una spirale a filo grosso in comunicazione colla pila, circondata da un altra a filo lungo e sottile congiunta ai capi del galvanometro. Per mezzo di bacchette di vetro poteva rimovere ed agitare la limatura. Mentre questo si faceva, l'ago del galvanometro rimaneva perfettamente a $0^{\circ}$. Comprimendo in vece la limatura, si avevano segni di una corrente che indicava aumento di magnetismo.

\section{Esperlenze sull' evaporazione dello zolfo a bassa temperatura.}

Una lamina di zolfo formata colando lo zolfo fuso in uno stampo di latta, era in uno dei scatfali di questo $I$. e R. Gabinetto appoggiata in una posizione inclinata sulla parete interna dello scaffale.

Questa lamina era ricoperta con una di gomma lacca. In questo stato rimasero le due lastre per tutta l' estate. La temperatura massima dell' estate decorso fu di $+31^{\circ}$ C. Osservate le lastre alla fine dell' estate, fu trovata tutta annerita la tavola di fondo dello scaffale e una sfumatura di questo colore esisteva all' intorno della lastra.

La tavola dello scaffale era stata verniciata con biacca e olio : per cui l' annerimento si spiega colla formazione del solfuro di piombo. Potrebbe credersi che il vapor aqueo e lo zolfo avessero potuto produrre l'idrogerte solforato: ma ciò che non era ad attendersi e che merita d'esser notato è lo strato di solfo in piccoli cristalli che esisteva sulla faccia della lamina di gomma lacca che era applicata sullo zolfo .

Credo perciò si debba d'ora innanzi ritenere che lo zolfo può volatilizzarsi ad una temperatura non maggiore di $+31^{0} \mathrm{C}$.

C. Matreveci. 